\title{
GROWTH OF COMPOSITE ENTIRE FUNCTIONS
}

\author{
By ANAND Prakash Singh
}

Introduction. If $f$ and $g$ are transcendental entire functions then Clunie [1] proved that $\lim _{r \rightarrow \infty} \frac{T(r, f(g))}{T(r, f)}=\infty$. An obvious question arises is, what can be said about the ratio

$$
\frac{\log T(r, f(g))}{T(r, f)}
$$

when $r \rightarrow \infty$ ? In general by considering $g(z)=e^{e^{z}}$, and $f(z)=e^{z}$, we see that the ratio (1) also tends to infinity. However if we put some restriction on the orders of $f$ and $g$ then we can show that the above ratio is bounded above by a finite quantity. Thus the purpose of this paper will be to prove some results dealing with the ratios that are of the form (1). We start with

THEOREM 1. Let $f(z)$ and $g(z)$ be entire functions of finte order such that $g(0)=0$ and $\rho_{g}<\lambda_{f} \leqq \rho_{f}$ where $\rho, \lambda$ denote respectively the order and the lower order for the corresponding functions. Then

$$
\limsup _{r \rightarrow \infty} \frac{\log T(r, f(g))}{T(r, f)} \leqq \rho_{f}
$$

Note. (i) From the hypothesis it is clear that $f$ must necessarily be transcendental.

(ii) The theorem does not hold true when $\rho_{g}=\rho_{f}$, for let $f(z)=e^{z}$ and $g(z)=e^{z}-1$, then $\rho_{g}=\rho_{f}=1$ and $T(r, f(g)) \sim \frac{e^{r}}{\left(2 \pi^{3} r\right)^{1 / 2}}$ see $[2,7]$, so that

$$
\limsup _{r \rightarrow \infty} \frac{\log T(r, f(g))}{T(r, f)}=\pi .
$$

(iii) In case $\rho_{g}>\rho_{f}$ we shall show that the limit superior will tend to infinity. Thus we shall prove

THEOREM 2. Let $f(z)$ and $g(z)$ be entire functions of finte order with $\rho_{g}>\rho_{f}$. Then

Received April 6, 1984

AMS (MOS) Classification No. 30D05, 30D20. 


$$
\limsup _{r \rightarrow \infty} \frac{\log T(r, f(g))}{T(r, f)}=\infty .
$$

For the proof of Theorem 1 we shall need the following lemma of K.! Niino and N. Suita [3].

Lemma. Let $f(z)$ and $g(z)$ be entire functions. Let $g(0)=0$. Then $T(r, f(g)$ ) $\leqq T(M(r, g), f)$ for all $r>0$.

Proof of theorem 1. By definition of order and lower order we have

$$
\begin{array}{ll}
T(r, f)<r^{\rho_{f}^{+\varepsilon}} & \text { for all } r \geqq r_{0} \\
T(r, f)>r^{\lambda_{f-s}} & \text { for all } r \geqq r_{0}
\end{array}
$$

$\left(r_{0}\right.$ need not be the same at every stage).

Now by the lemma

$$
\begin{aligned}
\log T(r, f(g)) & \leqq \log T(M(r, g), f) & & \\
& <\left(\rho_{f}+\varepsilon\right) \log (M(r, g)) & & \text { for all } r \geqq r_{0} \\
& <\left(\rho_{f}+\varepsilon\right) r^{\rho_{g}+\varepsilon} & & \text { for all } r \geqq r_{0} \\
& <\left(\rho_{f}+\varepsilon\right) r^{\lambda_{f}-\varepsilon} & & \text { by choosing }
\end{aligned}
$$

$\varepsilon>0$ so small that $\rho_{g}+\varepsilon<\lambda_{f}-\varepsilon$.

On the other hand, $T(r, f)>r^{2} f^{-\varepsilon}$ for all $r \geqq r_{0}$. Thus for large $r$,

$$
\frac{\log T(r, f(g))}{T(r, f)}<\left(\rho_{f}+\varepsilon\right) \text {. }
$$

The theorem now follows since $\varepsilon>0$ is arbitrary.

Proof of theorem 2. We prove this theorem on the same lines as K. Niino and C.C. Yang [4].

$$
\begin{aligned}
T(r, f(g)) & \geqq \frac{1}{3} \log M\left(\frac{1}{8} M\left(\frac{r}{4}, g\right)+o(1), f\right) & & \text { see }[4] . \\
& \geqq \frac{1}{3}\left\{\frac{1}{8} M\left(\frac{r}{4}, g\right)+o(1)^{\lambda_{f^{-s}}}\right. & & \text { for all } r \geqq r_{0} \\
& \geqq \frac{1}{3}\left\{\frac{1}{9} M\left(\frac{r}{4}, g\right)\right\}^{\lambda_{f}^{-s}} & & \text { for all } r \geqq r_{0} \\
& \geqq \frac{1}{3}\left(\frac{1}{9}\right)^{\lambda_{f^{-\varepsilon}}}\left\{\exp (r / 4)^{\left.\rho^{\rho^{-\varepsilon}}\right\}^{\lambda_{f-s}-s}}\right. & & \text { for a sequence }
\end{aligned}
$$

$r=r_{n} \rightarrow \infty$. Thus for a sequence $\left\{r_{n}\right\}$

$$
\log T\left(r_{n}, f(g)\right) \geqq \log A+\left(\lambda_{f}-\varepsilon\right)\left(r_{n} / 4\right)^{\rho g-\varepsilon}
$$


where $A=\frac{1}{3}\left(\frac{1}{9}\right)^{\lambda f^{-\varepsilon}}$.

On the other hand for all $r \geqq r_{0}, T(r, f)<r^{\rho_{f}+s}$. Thus for a sequence $\left\{r_{n}\right\}$ (where each $r_{n} \geqq r_{0}$ ) we have

$$
\frac{\log T\left(r_{n}, f(g)\right)}{T\left(r_{n}, f\right)}>\frac{\log A}{r_{n}{ }^{\rho_{f}+\varepsilon}}+\frac{\left(\lambda_{f}-\varepsilon\right)}{r_{n} \rho^{\rho^{+}+\varepsilon}}\left(\frac{r_{n}}{4}\right)^{\rho_{g}-\varepsilon} .
$$

And so, $\lim _{r \rightarrow \infty} \sup \frac{\log T(r, f(g))}{T(r, f)}=\infty$, since we can choose $\varepsilon>0$ such that $\rho_{g}-\varepsilon$ $>\rho_{f}+\varepsilon$. This proves theorem 2 .

An immediate consequence of theorem 1 is the following corollary

COROLLARY 1. Let $f$ and $g$ be entire functions satisfying the conditions of theorem 1. Further let $\lim _{r \rightarrow \infty} \inf \frac{\log T(r, f(g))}{T(r, f)} \geqq \rho_{f}$. Then the hyper order of $f(g)$ is $\rho_{f}$. (Hyper order of a function $f$ is defined to be $\limsup _{r \rightarrow \infty} \frac{\log \log T(r, f)}{\log r}$ ).

The proof follows easily since the hypothesis and the theorem 1 imply that $\log T(r, f(g)) \sim \rho_{f} T(r, f)$.

We now give an application of theorem 2 .

COROLLARY 2. Let $f$ and $g$ be transcendental entire functions of finite order. Further let $\rho_{g}>\rho_{f}$ then $f(g)$ is of infinite order.

$$
\text { Proof. } \begin{aligned}
\lim _{r \rightarrow \infty} \sup \frac{\log T(r, f(g))}{\log r} & =\lim _{r \rightarrow \infty} \sup \left\{\frac{\log T(r, f(g))}{T(r, f)} \cdot \frac{T(r, f)}{\log r}\right\} \\
& \geqq \limsup _{r \rightarrow \infty} \frac{\log T(r, f(g))}{T(r, f)} \lim _{r \rightarrow \infty} \inf \frac{T(r, f)}{\log r} .
\end{aligned}
$$

But for a transcendental entire function $f$, it is well known that $\lim _{r \rightarrow \infty} \frac{T(r, f)}{\log r}=\infty$. The result now follows using theorem 2 .

In [theorem 2,1], Clunie has proved that if $f$ and $g$ are transcendental entire functions then $\lim _{r \rightarrow \infty} \frac{T(r, f(g))}{T(r, g)}=\infty$. So the obvious question is what can be said about $\lim _{r \rightarrow \infty} \frac{\log T(r, f(g))}{T(r, g)}$ ? This we have been unable to solve. However if we consider the ratio $\frac{\log \log T(r, f(g))}{\log T(r, g)}$ or $\frac{\log T(r, f(g))}{\log T(r, g)}$ we have obtained the following two theorems.

THEOREM 3. Let $f$ and $g$ be transcendental entire functions of finte order. Let $g(0)=0$ and let $\lambda_{g}>0$. Then

$$
\lim _{r \rightarrow \infty} \sup \frac{\log \log T(r, f(g))}{\log T(r, g)} \leqq \frac{\rho_{g}}{\lambda_{g}} .
$$


Proof. As in theorem 1,

$$
\log T(r, f(g))<\left(\rho_{f}+\varepsilon\right) r^{\rho g+\varepsilon} \quad \text { for all } r \geqq r_{0} .
$$

Thus for all $r \geqq r_{0}$ we have

$$
\log \log T(r, f(g))<\log \left(\rho_{f}+\varepsilon\right)+\left(\rho_{g}+\varepsilon\right) \log r .
$$

On the other hand,

Thus

$$
\log T(r, g)>\left(\lambda_{g}-\varepsilon\right) \log r \quad \text { for all } r \geqq r_{0} .
$$

$$
\limsup _{r \rightarrow \infty} \frac{\log \log T(r, f(g))}{\log T(r, g)} \leqq \frac{\rho_{g}}{\lambda_{g}} .
$$

THEOREM 4. Let $f$ and $g$ be transcendental entire functions of finite order with $\rho_{g}>0$, then $\lim _{r \rightarrow \infty} \sup \frac{\log T(r, f(g))}{\log T(r, g)}=\infty$.

Proof. From (2), for a sequence $\left\{r_{n}\right\}$ we have,

$$
\log T(r, f(g)) \geqq \log A+\left(\lambda_{f}-\varepsilon\right)\left(\frac{r_{n}}{4}\right)^{\rho_{g}-\varepsilon}
$$

where $A=\frac{1}{3}\left(\frac{1}{9}\right)^{\lambda, f^{-\varepsilon}}$. Also

Thus

$$
\log T(r, g)<\left(\rho_{g}+\varepsilon\right) \log r \quad \text { for all } r \geqq r_{0} .
$$

$$
\frac{\log T\left(r_{n}, f(g)\right)}{\log T\left(r_{n}, g\right)} \geqq \frac{\log A}{\left(\rho_{g}+\varepsilon\right) \log r_{n}}+\frac{\lambda_{f-\varepsilon}}{4^{\rho g-\varepsilon}} \cdot \frac{\left(r_{n}\right)^{\rho g-\varepsilon}}{\left(\rho_{g}+\varepsilon\right) \log r_{n}}
$$

which tends to infinity as $r_{n} \rightarrow \infty$, since $\rho_{g}>0$. This yields the desired result.

\section{REFERENCES}

[1] Clunie, J., The composition of entire and meromorphic functions, Mathematical Essays dedicated to A. J. Macintyre (Ohio Univ. Press, 1970), 75-92.

[2] Hayman, W.K., Meromorphic functions (Oxford Univ. Press, 1964).

[3] Nino, K. And N. Suita, Growth of a composite function of entire functions, Koda1 Math. J., (3) 3 (1980), 374-379.

[4] Nino, K. ANd C. C. YANG, Some growth relationships on factors of two composite entire functions, Factorization theory of meromorphic functions and related topics, Marcel Dekker Inc. New York (1982), 95-99.

Department of Mathematics

ShIVAJi UNIVERSITY

KOLHAPUR-416004

(MAHARASHTRA)

INDIA 